\title{
Intuitive statistical inference: How pigeons categorize binomial samples
}

\author{
CHARLES P. SHIMP and FRANCES A. HIGHTOWER \\ University of Utah, Salt Lake City, Utah
}

\begin{abstract}
Pigeons categorized binomial samples produced by two complementary random processes. Samples were $1,2,4$, or 8 successively presented outcomes (vertical or horizontal lines) of, in effect, tossing one of two equally likely coins. One coin (A) was biased in favor of vertical, and the other coin (B) was similarly biased in favor of horizontal. Choosing red or green stimuli presented after each sample was reinforced with food if coin A or coin B had been tossed, respectively. In that sense, choosing red or green was logically analogous to inferring that the statistical evidence reflected tossing of coin $A$ or coin $B$, respectively. The statistical diagnosticity of a sample, the relative likelihood of its having been produced by a particular coin, equaled, except for sampling fluctuation, the relative frequency of reinforcement of a particular color given that sample, and was experimentally varied by the bias on the coins and by sample size. All the variables that affect optimal, formal inference about binomial samples also affected intuitive inference. But inferences were very suboptimal: "undermatching" was obtained in part due to control of categorization by the sequential structure of binomial samples. These results reveal limitations of optimality theories for animal decision making in the face of uncertainty when observations in samples are presented successively. On the other hand, they are generally compatible with molecular analyses of instrumental learning which assign an important role to the local temporal organization of events preceding reinforcement. Most generally, they show that maladaptive control over intuitive statistical inference by a variable upon which optimal performance does not depend is neither a uniquely human phenomenon nor dependent upon linguistic strategies.
\end{abstract}

We describe an experiment on "intuitive statistical inference," inference that does not explicitly involve formal computation. We asked two basic questions. First, can pigeons learn a task requiring intuitive statistical inference? Second, how adaptive is their performance on such a task in comparison with optimal, formal statistical influence?

An extraordinary range of phenomena can be interpreted in terms of intuitive statistical inference (Brunswick, 1939; Gigerenzer \& Murray, 1987; Staddon, 1988), but very little is known about the inductive acquisition of intuitive inference in extremely simple statistical tasks. We therefore adapted for this purpose a probabilistic discrimination, one in which the basis for discrimination between two situations is statistical: no feature or combination of features guarantees a correct identification of either situation (Estes, Burke, Atkinson, \& Frankmann, 1957). We used one of the simplest of all random processes, the Bernoulli-trials process (Feller, 1950), and required pigeons to learn to categorize the resulting binomial samples according to the processes that produced them. Thus, on any trial, an animal observed a binomial sample pro-

\footnotetext{
Data reported here were obtained by the second author in partial fulfillment of the requirements for a Master of Science degree. The first author is responsible for the present manuscript. The research was supported in part by a grant from the Biomedical Science Support Grant Committee at the University of Utah and by NIMH Grant R01 MH 42770. Correspondence should be addressed to C. P. Shimp, Department of Psychology, University of Utah, Salt Lake City, UT 84112.
}

duced by a sequence of outcomes of Bernoulli trials and then categorized the resulting sample. This categorization was logically equivalent to inferring which of the two complementary processes had produced the observed sample. The procedure worked as follows. Suppose one has two coins, one biased in favor of heads and the other biased to the complementary degree in favor of tails. One of the two coins is selected and then tossed some number of times. An observer tries to guess which coin was tossed and is afterwards given the correct answer. The observer is given many such samples. We asked if pigeons could learn such a problem and, if so, how their categorizations of binomial samples compared to optimal categorization.

Optimal inference would consist here of calculating for each observed sample the relative likelihood that it was produced by one random process rather than the other, and then categorizing the sample in terms of the more likely one. In binomial tasks such as the present one, this likelihood ratio upon which optimal inference depends involves only the bias on the coins, the amount of data or sample size (i.e., the number of tosses of a coin), and the base rate probability of a particular coin's being chosen to be tossed. We will call the relative likelihood ratio of sample its diagnosticity. A binomial sample's diagnosticity does not depend on its sequential structure of heads and tails, only on the number of heads and tails, since observations in a sample are independent.

To review, we investigated "intuitive" statistical inference with one of the simplest possible sampling proce- 
dures, a binomial arrangement in which, in effect, a subject was shown some evidence logically equivalent to outcomes of a number of tosses of a biased coin, and then asked to categorize the sample in a manner logically equivalent to inferring which of two coins was tossed. Correct, reinforced categorizations were those which corresponded to the actual processes that produced the samples.

We varied two experimental parameters that affect the statistical diagnosticity of binomial samples and hence optimal statistical inference, the probability that any single observation in a sample diagnosed a particular underlying process and sample size.

\section{METHOD}

\section{Subjects}

Subjects were eight White Carneaux pigeons (Columba livia) which had served previously in various short-term memory experiments. Bird 2 had, in addition, most recently served in an experiment using a pigeon chamber with a CRT display and an infrared response-detection system instead of the usual pecking keys used here (Clauson, Izatt, \& Shimp, 1985). The pigeons were maintained at $80 \%$ of their free-feeding weights, $\pm 10 / \mathrm{g}$. Grit and water were always available in their home cages, where there was a $14: 10-\mathrm{h}$ light:dark cycle.

\section{Apparatus \\ Three standard three-key Lehigh Valley Electronics pigeon cham- bers were interfaced with a PDP-8/E computer which arranged all experimental events and recorded the data. Ventilator fans and white noise helped to mask extraneous sounds.}

\section{Procedure}

The experimental procedure is conveniently viewed in terms of a coin-tossing interpretation of Bernoulli-trials processes. On each of a series of trials, a subject in effect saw a sample of heads and tails obtained by $1,2,4$, or 8 tosses of one of two coins. The coin tossed for any given sample was selected randomly. Coin A had probability of tails $P_{\mathrm{A}}$, and coin B had the complementary probability of tails $P_{\mathrm{B}}=1-P_{\mathrm{A}}$. The value of $P_{\mathrm{A}}$ was always greater than .5 so that coin $A$ was always biased in favor of tails and coin $B$ was always biased in favor of heads. Coins $A$ and $B$ were equally likely to be tossed $1,2,4$, or 8 times. After observing all the outcomes of the $1,2,4$, or 8 tosses on a trial, a subject was in effect asked which coin was tossed, in the sense that if coin $A$ were tossed, one response was correct, a response to a red stimulus, and if coin B were tossed, another response was correct, a response to a green stimulus. It will be convenient to speak of vertical-biased coin $\mathrm{A}$, when a choice of red was correct, or horizontal-biased coin B, when a choice of green was correct. Thus, it will also be convenient to speak of the "vertical-biased red choice" and the "horizontal-biased green choice."

A session lasted $1 \mathrm{~h}$ and was a series of discrete trials. Each trial consisted of a study component, a test component, and if necessary, a correction component. Each of these components is described below in detail.

\section{Study Component}

The study component involved successive presentations of the stimuli (heads or tails) on a center key. The two stimuli representing the outcomes of tossing coin A or coin B were a white vertical line on a blue background (a "tail") and a white horizontal line on a dark background (a "head"), respectively. The computer chose, with probability .5 , which coin to toss on each trial. A trial began with the presentation on the center key of the stimulus representing the outcome of the first toss of coin A or coin $B$. The stimulus remained on the center key until a subject responded to it after a minimum observation time of $.5 \mathrm{sec}$. (That is, there was a fixed-interval .5-sec schedule arranged for terminating an observation.) A response to the center key after $.5 \mathrm{sec}$ either terminated the study component, if the sample size for the trial was 1 , or started an interobservation interval of $.25 \mathrm{sec}$ if the sample size was greater than 1. During the interval, all lights were off. If the sample size was 2 or greater, the stimulus representing the outcome of the second toss of the same coin was then presented on the center key. This second observation, like all others, remained on until the subject responded to the center key after a minimum of $.5 \mathrm{sec}$. Note that the probability of a head was constant throughout a trial, since the same coin was tossed for every observation in a sample. The number of observations in any sample was $1,2,4$, or 8 , and, as noted above, all four possible sample sizes were equally likely.

\section{Test Component}

The termination of the last observation in a sample initiated a .1-sec blackout. Then two side keys appeared, red and green. The side on which a particular color appeared varied randomly. A subject's task was to respond to the color corresponding to the random process that had produced the preceding sample: Its task may be interpreted to have been to respond to the color that represented the coin tossed on that trial, red for tails-(vertical)-biased coin A and green for heads-(horizontal)-biased coin B. If a subject responded to the color corresponding to the coin tossed, the lights went out and a reinforcer (mixed grain) was delivered for $1.5 \mathrm{sec}$. An intertrial interval of $40 \mathrm{sec}$ then elapsed before the beginning of the next trial.

\section{Correction Component}

A response to the incorrect side key began a correction interval, a 5-sec blackout, after which the same sequence of observations was repeated again. This same sample was repeated after any subsequent error until a subject made the correct response (the position of which stayed the same) and a reinforcer was delivered.

\section{Pretraining}

Total pretraining lasted for approximately 5 months while experimental parameters were selected. The stimuli used initially were a white horizontal or vertical line against a dark background. A blue background was later added to the vertical line to facilitate discrimination of heads and tails. The experimental parameters varied during pretraining included sample size, observation duration, intertrial interval, and the bias on coins $A$ and $B$. At the end of pretraining, sample size was 8 and $P_{\mathrm{A}}$ was 1.0 . Otherwise, the terminal pretraining conditions were as described above for the experiment proper. Pretraining ended when a bird discriminated white vertical lines against blue from horizontal lines against a dark background with a minimum accuracy of $90 \%$.

$P_{\mathrm{A}}$ was decreased from .9 to .6 by .1 increments during the first four experimental conditions. $P_{\mathrm{A}}$ was set equal to .9 during the fifth phase of the experiment. The final two conditions of the experiment were run following an interruption of several months. During Conditions 6 and $7, P_{\mathrm{A}}$ was equal to .9 and .6 , respectively. Each condition lasted 30 sessions. The average number of trials per session was 69 (averaged across the last 5 days of all conditions and across all 8 pigeons). The range of the average number of trials per day across conditions was 65 to 71 .

\section{RESULTS}

We sought to determine if pigeons could learn a task requiring intuitive statistical inference. Thus, we asked if statistical diagnosticity affected pigeons' categorizations 


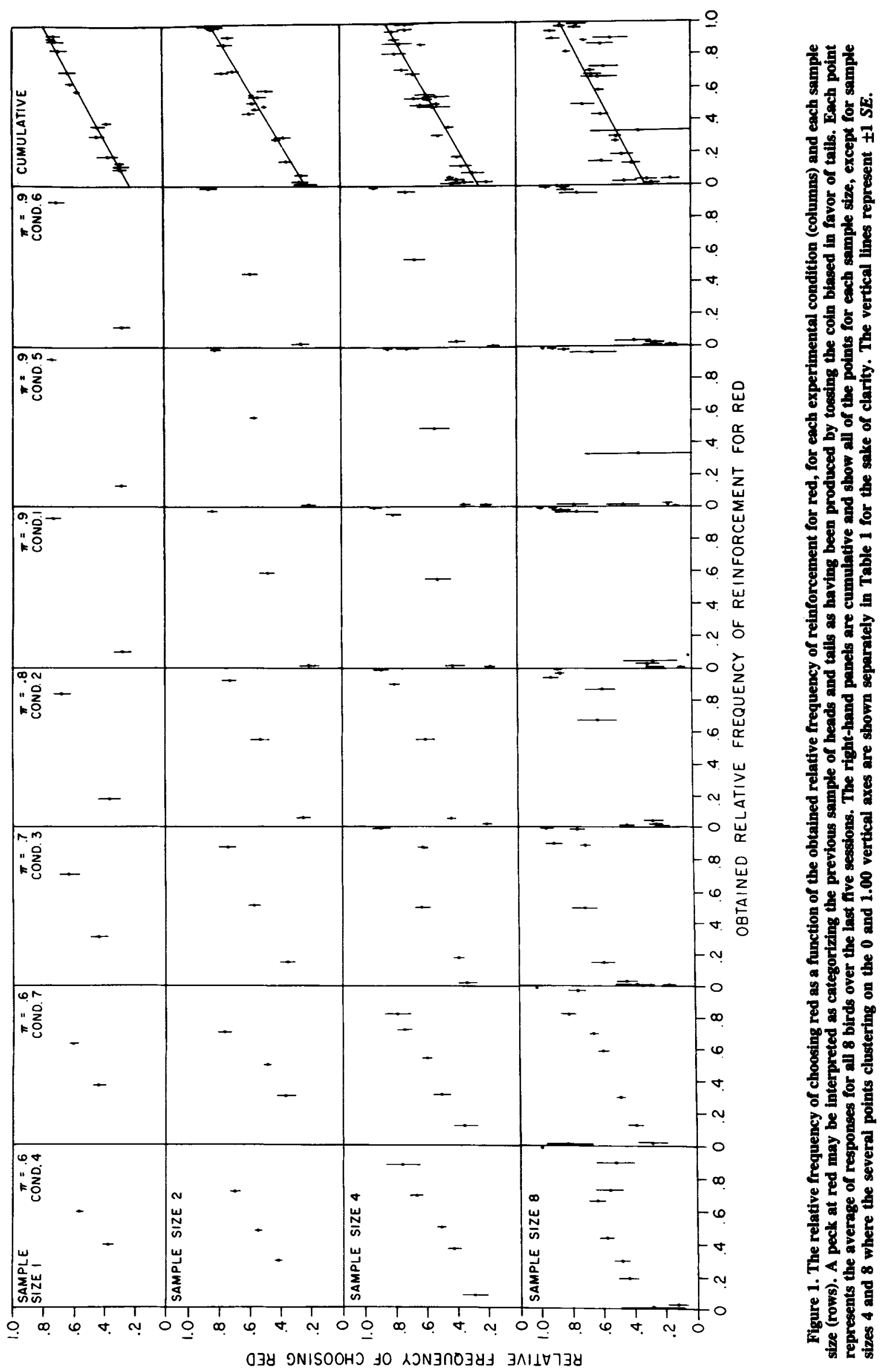


of ambiguous samples. The appropriate dependent variable is the relative frequency of choosing red after a particular sample of heads and tails. This is the relative frequency of categorizing a sample as diagnosing or belonging to the "vertical-biased" or "tails-biased" category A. It was calculated for each of the 8 birds, and then these eight relative frequencies were averaged. All data presented here are in terms of these group averages. The vertical lines in each figure represent $\pm 1 S E$ and reflect variability across subjects, that is, across the eight individualsubject 5-day averages. Responses counted in the calculations include only the first choice on each trial: they do not include any responses during correction components.

The independent variable in Figure 1 is the obtained relative frequency of reinforcement for the red choice given a particular sample, or the number of reinforced pecks at red divided by the total number of reinforced pecks at red and green. Subjects' choices did not affect this variable. It depended only on the sampling characteristics.

In view of the task as one involving intuitive inference, we may say Figure 1 shows the relative frequency of categorizing a sample as one produced by random process $\mathrm{A}$ as a function of the obtained relative frequency with which that sample actually was produced by process $A$. More succinctly, Figure 1 shows how intuitive inference depended on statistical diagnosticity. Each point in the figure represents a different type of sample with a specific number of observations and a specific number of heads. For example, for sample size 2, there were three possible types of samples: no heads and two tails, one head and one tail,

Table 1

Choice Behavior on Trials

with Extreme Diagnosticities: Obtained Relative Frequency of Reinforcement for the Tails-Biased Red Choice*

\begin{tabular}{|c|c|c|c|c|}
\hline $\begin{array}{l}\text { Condition } \\
\text { Number }\end{array}$ & $P_{\mathrm{A}}$ & $\begin{array}{l}\text { Number } \\
\text { of Tails }\end{array}$ & $\begin{array}{l}\text { Relative } \\
\text { likelihood the } \\
\text { sample diagnosed } \\
\text { tails-biased } \\
\text { random } \\
\text { process } A\end{array}$ & $\begin{array}{l}\text { Average relative } \\
\text { frequency } \\
\text { of categorizing } \\
\text { the sample as } \\
\text { diagnosing } \\
\text { process A }\end{array}$ \\
\hline
\end{tabular}

$\begin{array}{llll}\text { Number } & P_{\mathrm{A}} \text { of Tails } & \text { process A }\end{array}$

$\begin{array}{ll}1,5,6 & .9 \\ 2 & .8 \\ 3 & .7 \\ 1,5,6 & .9 \\ 2 & .8 \\ 3 & .7 \\ 1,5,6 & .9 \\ 2 & .8\end{array}$

0
0
0
1
1
1
2
3

$.00 \quad .09$

$.00 \quad .19$

$.00 \quad .18$

$.00 \quad .20$

$.00 \quad .23$

$.01 \quad .37$

$.00 \quad .31$

$.06 \quad .41$

Relative Frequency of Reinforcement $=1.0$

\begin{tabular}{lllll}
$1,5,6$ & .9 & 6 & 1.00 & .82 \\
$1,5,6$ & .9 & 7 & 1.00 & .87 \\
2 & .7 & 7 & 1.00 & .86 \\
3 & .7 & 7 & 1.00 & .94 \\
$1,5,6$ & .9 & 8 & 1.00 & .95 \\
\hline
\end{tabular}

* Relative likelihoods of 8-observation samples diagnosing tails-biased random process $A$ and average relative frequencies of categorizing those samples as diagnosing random process $A$, for cases with obtained relative frequencies of reinforcement of 0 or 1 . or two heads and no tails. The relative frequencies of choosing red after these three types of samples are represented by the three points in the next-to-the-top row. The right-hand panels are cumulative and show for each sample size all of the points from the other panels in a given row-that is, all the points from the seven conditions for that sample size except for sample sizes 4 and 8 , where the several points that cluster tightly on the 0 or 1.00 vertical axes are omitted simply for the sake of clarity, and are shown separately in Table 1 .

A glance at Figure 1 is sufficient to answer our first question: pigeons clearly can learn a task involving intuitive statistical inference. Consider the cumulative panels of the figure. The equations for the best-fitting straight lines for sample sizes of $1,2,4$, and 8 have $y$-intercepts of $.24, .23, .27$, and .29 , and corresponding slopes of $.54, .62, .56$, and .55 , respectively. Neither slopes nor $y$-intercepts vary much across sample size. The resulting similarity in these functions suggests that statistical diagnosticity was an important determiner of categorization of ambiguous samples. On the average, different samples with different sizes and different numbers of heads and tails, but with similar diagnosticities, were categorized fairly similarly: pigeons' categorizations of statistically ambiguous samples indicated sensitivity to their statistical diagnosticities.

Now let us compare performance to optimal performance, which was always to categorize a sample in terms of whichever was the relatively more likely category. In Figure 1, this would produce a step function rising from 0 to 1 at a diagnosticity of 0.5 . Although pigeons clearly learned to categorize ambiguous samples in a way that depended on their statistical properties, performance was far from optimal. The obtained functions show "undermatching" because the relative frequency of categorizing a sample as an instance of category A deviated from the corresponding relative frequency of reinforcement in the direction of indifference.

Figure 2 shows the relative frequency of choosing red as a function of the two variables that affect diagnosticity, sample size and sample composition. The top row shows this function for the most diagnostic samples, those in which all of the observations were heads or tails. For such examples, the relative frequency of choosing red generally increased or decreased with sample size when all the observations were tails or heads, respectively. The two curves in each panel generally diverge, with the difference between the relative frequency of choosing red for all heads and for all tails getting larger as the sample size increases. This divergence indicates that the birds did not just look at one or two observations and ignore the rest. The second row of panels shows the data for the next most diagnostic type of sample, composed of one head with all the other observations tails or composed of one tail with all the others heads. The relative frequency of the tails-biased red choice increased when there was one head and increasing numbers of tails, and decreased when there was one tail and increasing number of heads. Again, 


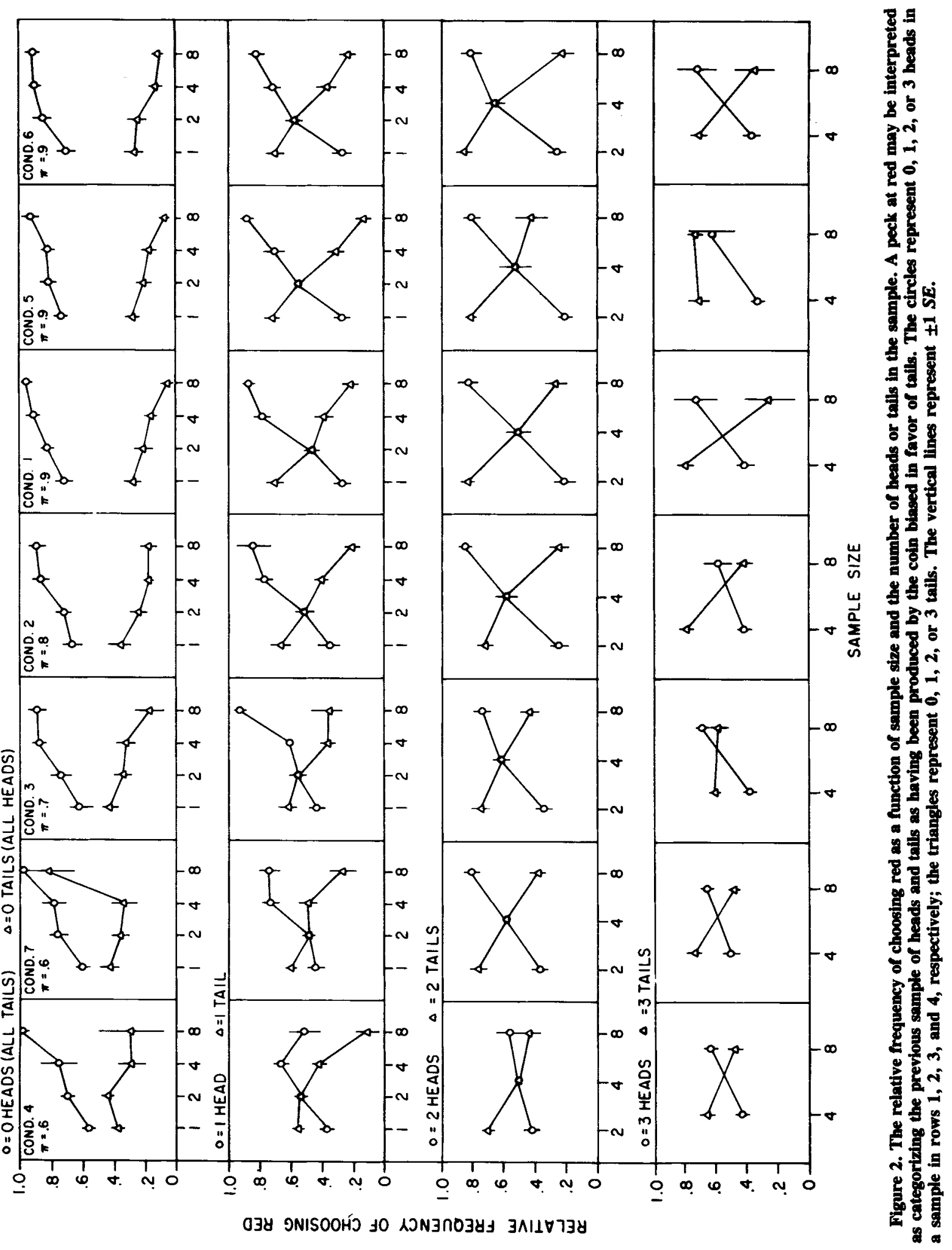




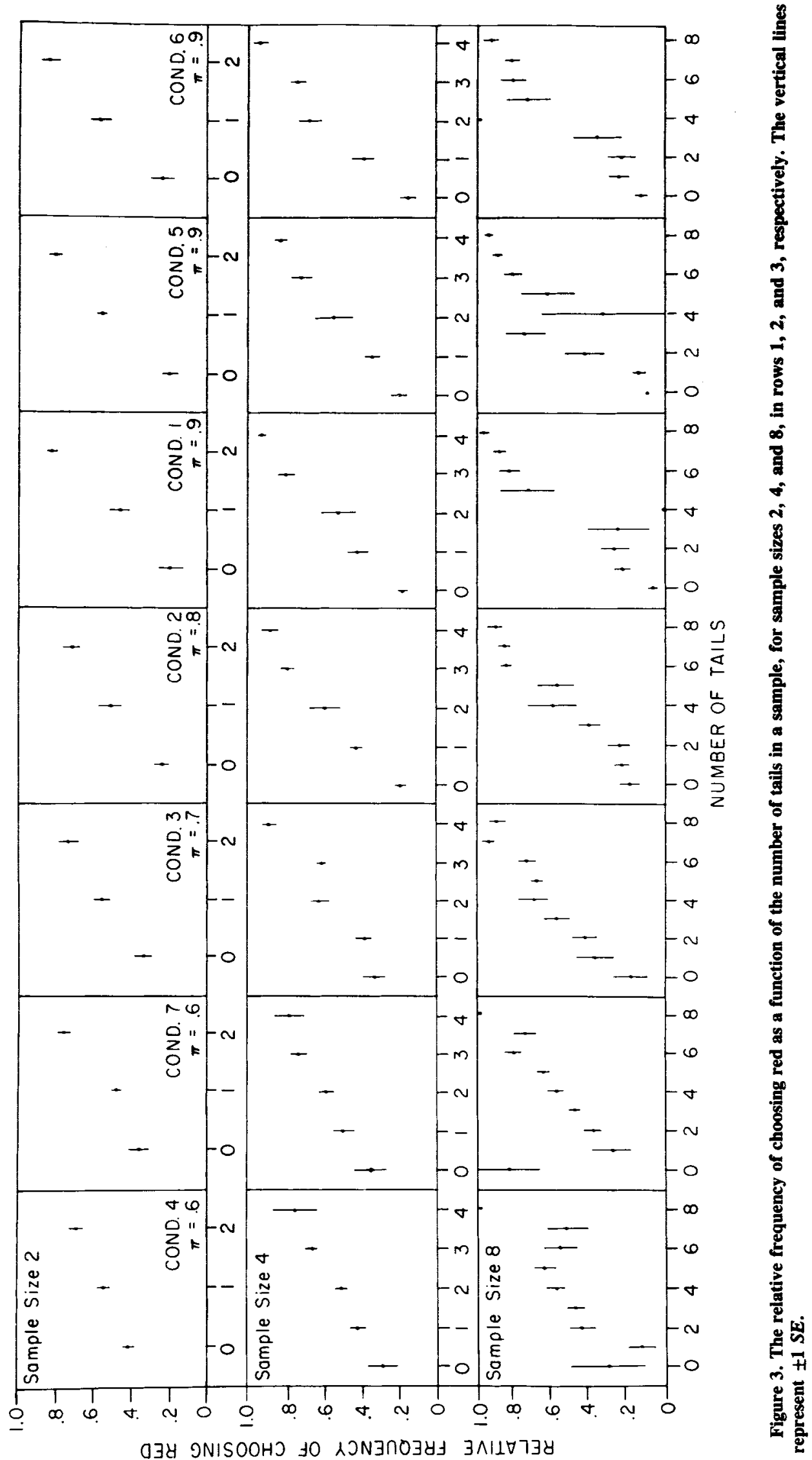




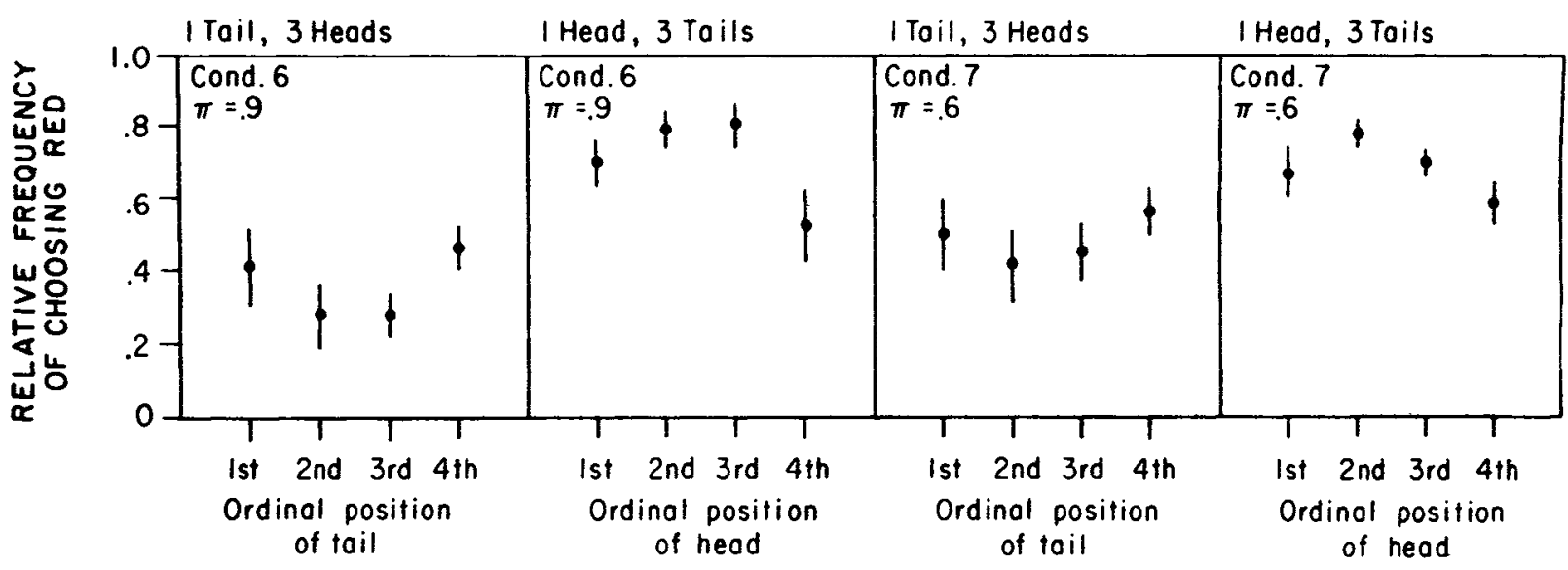

Figure 4. The relative frequency of choosing red as a function of the ordinal position of heads and taik, for Conditions 6 and 7 , sample size 4.

categorizing a sample in a particular way was an increasing function of the relative likelihood that the sample really did indicate that category. The other rows in Figure 2 show similar effects for other types of samples which were still less diagnostic. For example, the bottom row shows the effect of sample size when there were three heads or three tails in a sample. The theoretical probabilities of three heads in a sample of 4 and of five heads in a sample of 8 are identical to the third decimal place. That is, in this case, larger samples were not necessarily more diagnostic, and accordingly, the two functions do not differ as much as in the other rows.

Figure 3 shows much of the same information as Figure 1, but plotted in terms of a different horizontal coordinate. It shows very clearly that the relative frequency of the tails-biased red choice increased as a function of the number of tails.

The sequential positions of heads and tails on each trial were recorded in Conditions 6 and 7 for samples with four features. Because of practical limitations, including the small size of the computer memory, these sequential data for samples of size 8 were not recorded. Figure 4 shows the relative frequency of the tails-biased red choice as a function of the location of one head in samples with three tails, or of one tail in samples with three heads. The samples represented in the left panel of Figure 4, for example, consisted of one tail and three heads. The first point in the panel represents the sequence tail, head, head, head; the second point represents the sequence head, tail, head, head; the third, the sequence head, head, tail, head; and the last sequence head, head, head, tail. It was difficult to judge the reliability of sequential effects based on five sessions, so Figure 4 shows averages over the last 10 sessions of each condition. The figure suggests that categorization depended more on the first and last observations than on the middle ones. To see this, note that the probability of the tails-biased red choice, the nonoptimal strategy for samples with three heads and one tail, is a U-shaped function of the serial position of the one tail; the probability of maladaptively choosing red increased when the one tail was first or last in the sample of four observations. The corresponding effect can also be seen in the other two panels, where the function is an inverted $U$.

The serial position effect was obviously reliable, even by a sign test derived from (appropriately enough) a binomial distribution: If each difference in relative frequency of choosing red between first and second positions or between third and fourth is scored as a + or as a - according to whether it was in the appropriate direction for primacy or recency, respectively, then we see that the probability of getting the observed result by chance is highly unlikely $\left(2^{-8}\right)$, since all eight differences were in the right direction.

Figure 4 shows that when observations in ambiguous samples were presented successively, categorization depended on more than diagnosticity: Different samples with the same diagnosticity were categorized differently depending on their sequential organization. This difference seems attributable to the consequences of short-term forgetting, where primacy and recency effects would produce better memory for first and last items in a list and, therefore, more effective use of information associated with them.

\section{DISCUSSION}

We developed a method that permits the study of "intuitive" statistical inference in infrahumans. A subject was repeatedly shown samples from two random processes and was asked to categorize each sample. The correct, reinforced categorization of a sample was that which corresponded to the process that actually produced it. Stated differently, a subject had to learn inductively from experience with reinforced ambiguous samples how to discriminate between two different random processes. We were interested in seeing how this intuitive statistical inference qualitatively compared with optimal statistical in- 
ference when the underlying random processes were Bernoulli-trials processes. In this case of binomial samples from two equally likely processes, optimal inference depends only on the bias of the two processes, which may be thought of as the bias towards heads or tails of two repeatedly tossed coins, the amount of data (number of coin tosses) in a sample, and the number of tails in a sample. These variables permit the computation of a sample's diagnosticity, or the relative likelihood of its having been produced by a particular random process. The optimal inference is always, of course, to categorize a sample as corresponding to the random process having the greater relative likelihood.

We found that each of these variables which determines optimal, formal statistical inference also plays a role in intuitive statistical inference: each affected how pigeons categorized binomial samples. However, the degree to which these variables determined inference was very suboptimal: the relative frequency with which a sample was categorized in a particular way revealed striking insensitivity to its statistical diagnosticity. One form of suboptimal behavior has been called "probability matching" in tasks involving probabilistically reinforced choice behavior (Estes, 1972). Probability matching would have been obtained here had the relative frequency of categorizing a sample in a particular way approximately equaled the probability that the sample actually had been from that category. The degree of insensitivity to statistical diagnosticity obtained here was even less than that corresponding to probability matching: we obtained undermatching. The literature on discrete-trials, probabilistically reinforced choice behavior in tasks with fixed reinforcement probabilities for specified stimuli has focused chiefly on degrees of adaptation ranging between matching and maximizing (Graf, Bullock, \& Bitterman, 1964; Hale \& Shimp, 1975; Shimp, 1966, 1973), but the present results remind us that any general theory will have to deal with discrete-trials behavior that is adaptive yet suboptimal to a degree even below that of probability matching.

Two methodological differences between the present experiment and previous ones appear likely to be responsible for the reduced sensitivity to statistical diagnosticity obtained here. Here, every day birds saw many different kinds of samples with different diagnosicities, whereas in previous probability-learning experiments they have seen only a few. Also, here component observations in a sample were presented sequentially, one at a time, whereas in a previous probabilistic discrimination components of a stimulus configuration were presented simultaneously (Shimp, 1973). Sequential observations, however common they may be in naturalistic settings, would seem inevitably to produce some forgetting, with a consequent loss of diagnostic information. Optimal use of information in a binomial samples does not depend on whether observations in the sample are presented sequentially or simultaneously. We therefore see an important difference here between formal and informal statistical inference. Although all the task variables that affect the former also affect the latter, some variables affect intuitive inference that do not affect optimal inference.

In particular, because of the serial position effect in short-term forgetting (see Figure 4 above and, e.g., Sands, Urcuioli, Wright, \& Santiago, 1984; Terrace, 1986), the way different sequential observations in a sample contribute to overall categorization is presumably complex: first and last observations contribute more than those nearer the middle. Thus, we see here a situation in which the optimal use of information required a subject's performance not to depend on sequential organization of recent environmental events, but nevertheless this local organization did acquire control of behavior. Local patterning of recent events controlled behavior despite the maladaptive nature of this control.

This control by the sequential organization of events is interesting in two ways. First, it demonstrates control of intuitive statistical inference by inappropriate features of samples. With human subjects, this result is commonly attributed to a subject's misinterpretation or misuse of linguistic knowledge (Kahneman, Slovic, \& Tversky, 1982; Nisbet, Krantz, Jepson, \& Kunda, 1983). We have therefore shown here that the dependency of intuitive statistical inference on inappropriate sampling variables is neither a uniquely human effect nor necessarily dependent on language.

Control of behavior by the sequential organization of previous events is interesting also in the light of the metatheoretical basis for a molecular analysis of behavior, where it is assumed that reinforcement tends to impose local temporal organization on behavior output because reinforcement operates on a pattern of events a subject remembers having recently experienced. Under most reinforcement contingencies, this produces adaptive behavior, such as in interresponse time contingencies, or at least not maladaptive behavior, such as in classical variableinterval and variable-ratio contingencies (Shimp, 1978, 1984). The present results show that reinforcement's effects may depend on the temporal organization of previous events even if the result is less, rather than more, adaptive behavior-less adaptive, that is, than if reinforcement's effects were limited to behavior contiguous with it or to behavior preceding it without regard to its sequential organization (as in Catania, 1971). Thus, the present results clearly show that it is not necessary to require control by recent patterns of events for those patterns to acquire control.

The study of intuitive statistical inference in infrahumans is in a very early stage of theoretical development. Nevertheless, it is already possible to see that several major categories of theories of human statistical inference will not generalize to infrahumans, at least not as they have been articulated so far. Most theories attribute inference to linguistic knowledge or strategies and are therefore obviously inadequate. Some do not address the issue of how inference is inductively learned, only how it is used in one-time categorical judgments. It is unclear how these theories could apply to the present data. Statisti- 
cal learning theory, while applicable to nonlinguistic settings like the present ones, confronts a nontrivial problem in the form of the present Figure 4, showing both primacy and recency within a single trial. The degree of adaptation displayed by animals, from undermatching to overmatching, also represents a challenge for quantitative theory. Despite all these difficulties, attempts to construct a quantitative model might prove worthwhile because of the important role attributed to inference in general accounts of learned behavior (Gigerenzer \& Murray, 1987; Staddon, 1988).

\section{REFERENCES}

Brunswick, E. (1939). Probability as a determiner of rat behavior. Journal of Experimental Psychology, 25, 175-197.

CATANIA, A. C. (1971). Reinforcement schedules: The role of responses preceding the one that produces the reinforcer. Journal of the Experimental Analysis of Behavior, 15, 271-287.

Clauson, H. D., Izatt, E. J., \& Shimp, C. P. (1985). An infrared system for the detection of a pigeon's pecks at alphanumeric characters on a TV screen: The dependency of letter detection on the predictability of one letter by another. Journal of the Experimental Analysis of Behavior, 2, 257-264.

EsTES, W. K. (1972). Research and theory on the learning of probabilities. Journal of the American Statistical Association, 67, 81-102.

Estes, W. K., Burke, C. J., Atkinson, R. C., \& Frankmann, J. P. (1957). Probabilistic discrimination learning. Journal of Experimental Psychology, 54, 233-239.

FELLER, W. (1950). An introduction to probability theory and its applications (Vol. 1). New York: Wiley.

Gigerenzer, G., \& MurRay, D. J. (1987). Cognition as intuitive statistics. Hillsdale, NJ: Erlbaum.
Graf, V., Bullock, D. H., \& Bitterman, M. E. (1964). Further experiments on probability-matching in the pigeon. Journal of the Experimental Analysis of Behavior, 7, 151-157.

HALE, J. M., \& ShImP, C. P. (1975). Molecular contingencies: Reinforcement probability. Journal of the Experimental Analysis of $\mathrm{Be}$ havior, 24, 315-321.

Kahneman, D., Slovic, P., Tversky, A. (1982). Judgment under uncertainty: Heuristics and biases. New York: Cambridge University Press.

Nisbet, R. E., Krantz, D. H., Jepson, C., \& Kunda, Z. (1983). The use of statistical heuristics in everyday inductive reasoning. Psychological Review, 90, 339-363.

Sands, S. F., Urcuioli, P. J., Wright, A. A., \&antiago, H. C. (1984). Serial position effects and rehearsal in primate visual memory. In H. L. Roitblat, T. G. Bever, \& H. S. Terrace (Eds.), Animal cognition (pp. 375-388). Hillsdale, NJ: Erlbaum.

ShImP, C. P. (1966). Probabilistically reinforced choice behavior in pigeons. Joumal of the Experimental Analysis of Behavior, 9, 443-455.

Shimp, C. P. (1973). Probabilistic discrimination learning in the pigeon. Journal of Experimental Psychology, 87, 292-304.

ShIMP, C. P. (1978). Memory, temporal discrimination, and learned structure in behavior. In G. H. Bower (Ed.), The psychology of learning and motivation (Vol. 12, pp. 39-76). New York: Academic Press. Shimp, C. P. (1984). Relations between memory and operant behavior, according to an associative learner (AL). Canadian Journal of Psychology, 38, 269-284.

STaddon, J. E. R. (1988). Leaming as inference. In R. C. Bolles \& M. D. Beecher (Eds.), Evolution and leaming (pp. 59-77). Hillsdale, NJ: Erlbaum.

TERRACE, H. S. (1986). A nonverbal organism's knowledge of ordinal position in a serial learning task. Journal of Experimental Psychology: Animal Behavior Processes, 12, 203-214.

(Manuscript received December 22, 1989; revision accepted for publication June 18,1990 .) 